\title{
The Slaw and the Slow Cooked: Culture and Barbecue in the Mid-South
}

\author{
James R. Veteto and Edward M. Maclin, eds. 2011 Vanderbilt University Press, Nashville. Pp. $216 \$ 22.95$ \\ (paperback). ISBN 978-0826518019
}

Reviewed by Jon D. Holtzman

Reviewer address: Department of Anthropology, Western Michigan University, 1903 W Michigan Ave, Kalamazoo MI 490085306. jon.holtzman@wmich.edu

The authors of this volume love barbecue. Seemingly every kind of barbecue and from a broad assortment of analytical frameworks and perspectives. Mostly hog, slow smoked, but they'll take it on the bone, chopped, minced, pulled, shredded, chipped and much more, on a plate or on a bun, with sauce or "dip" ranging from vinegar with nothing more than flakes of pepper, to mustard or mayonnaise based sauces, to thick, sweet ketchupy ones, sometimes but not only including what Veteto and Maclin maintain is fundamental to a good Mid-South barbecue sauce-some combination of vinegar, tomato, pepper and a sweetener. This is not a disinterested text. While rigorously scholarly, taking perspectives centered mainly but not exclusively on anthropology, the editors and contributors are passionate about their hog, particularly the varieties prepared in ways rooted in their own upbringings in the Mid-South.

The Slaw and the Slow Cooked is composed of eleven chapters: a theoretical introduction and ten chapters focusing on varied aspects of time-honored and contemporary barbecue. Although the book is divided into two sections- "Traditional and Contemporary Landscapes of Mid-South Barbecue" and "Old New Barbecue Moving Forward"- the editors note that the divide between them is fairly arbitrary, and indeed it is not entirely clear what contrast between the halves was intended. This minor organizational issue aside, the chapters are varied, solid and engaging. The editors are both anthropologists, and, while the book has a strong anthropological tilt, its contributors also include scholars in literature and folklore, food writers, chefs, and historians. The authors have a number of clear aims and address them effectively. They endeavor to make a firm contribution to the anthropology of food, to some extent theoretically but perhaps more so empirically.
Veteto and Maclin emphasize the importance of barbecue as a central vehicle of both identity and difference in the American South. On one hand, the authors argue it is such a key lived symbol and experience within Southern life that (following Southern sociologist John Shelton Reed) a flag emblazoned with a dancing pig with a fork and knife might rightly replace the racism tainted flag of the Confederacy as the banner of the South. On the other hand, however, regional and local differences in how to prepare hog form fundamental bases for distinctions between communities, such that "...most Southerners who engage in the act of cooking or consuming barbecue think that the way they are used to having their barbecue prepared is the only way God intended it to be done" (p. 8). Along with the analytical focus on identity and difference, Veteto and Maclin's introduction also raises themes concerning race, gender, and memory in regard to food. In these varied areas the volume does not necessarily break new theoretical ground, but certainly outlines in detail barbecue's rightful place as an excellent phenomenon to explore these scholarly concerns. This fits extremely well with other goals of the volume, namely to make scholarly anthropological writing more engaging and accessible through attention to a topic of broad culinary interest, as well as to make food writing more scholarly through cross-fertilization with rigorous intellectual concerns.

Most of the chapters engage with barbecue with quite in-depth, specific case studies, although these are effectively framed by those that give a broader perspective on topics such as the history of barbecue and the relation of barbecue to concerns such as tradition in the digital age and food sustainability, with the latter tied to issues such as the Slow Food Movement and heritage varieties of pigs and toma- 
toes. The case studies tend toward the introduction's emphasis on identity and difference, but approach these topics in a wide range of ways. Several deal with specific producers of barbecue, some with restaurants that are particularly notable or well-known in the micro-region upon which the author focuses, and some with detailed analysis of a geographically focused form of barbecue tradition. These examples do not focus only on the food per se, but show how broader issues such as gender relations and historical and contemporary racism may be enacted through food practices. Some of the chapters emphasize the social processes through which the food is produced-whether in a context as commonplace as a charity picnic, or in the highly specialized arena of hog roasting competitions-while many focus more heavily on the nature of the food itself. Pervasive throughout most of these pieces is an emphasis on tradition, though this is displayed not to be timeless and unchanging with attention to how it has developed over time and how it may evolve in the future.

If there is a drawback to this book it lies in its qualities that are also its strength. The editors and contributors all exude a tremendous enthusiasm in their work and their subject matter, for barbecue in general and for the particular versions of it that are beloved from their communities or childhoods, making this an engaging, readable, and surprisingly personal book. The other side of the hog in this, however, is that I found it at times difficult to separate the authors' passion for barbecue from the empirical importance that they are attributing to their subject matter. Does everyone in the mid-South actually share the beliefs and attitudes that are both pervasive, and portrayed as essentially universal in this text? This is an issue that readers should consider when engaging with the text, but a fairly minor one. The bottom line is that the authors are having fun, but serious fun, and the book is better for it. There is much to be learned from this book in regard to the anthropology of food, the ethnography of the U.S. and, of course, barbecue. The book is highly scholarly, appropriate for engaging students in the classroom, and accessible for lay readers interested in foodways. I highly recommend it for anyone interested in the anthropology of food. 